\title{
A Monte-Carlo method used to study the fragment impact effect on the industrial facilities
}

\author{
Q.B. Nguyen ${ }^{1,2, a}$, A. Mebarki ${ }^{1}$, R. Ami Saada ${ }^{1}$, F. Mercier $^{2}$ and M. Reimeringer ${ }^{2}$ \\ 1 Université Paris-Est, Laboratoire de Modélisation et Simulation Multi-échelle, MSME FRE 3160 CNRS, 5 boulevard Descartes, \\ 77454 Marne la Vallée, France \\ ${ }^{2}$ Institut National de l'Environnement Industriel et des Risques (INERIS), 10 boulevard Lahitolle, 18000 Bourges, France
}

Received 19 December 2007, accepted 27 March 2008

\begin{abstract}
The generated fragments due to the explosion of vessel under pressure may be threats to the surrounding equipments at the impact. The fragment characteristics are number, shape, mass, departure angles, departure velocity. These characteristics are considered as uncertain variables. The authors propose the probability distribution for each variable. A fragment trajectory model concerning its shape is then presented in order to study the impact problem. The numerical simulations using the Monte-Carlo method allow calculating the probability of impact, $P_{i m p}$ between fragments and surrounding equipments.
\end{abstract}

Key words: Explosion; vessel; fragment; source term; trajectory; impact; probability.

\section{Introduction}

The industrial site shelters cylindrical vessels under pressure of gas or liquids. This is a closed system. The overpressure or the fragment aggression is the cause of the vessel explosions. The fragment series can be generated after an explosion. Nevertheless, all the generated fragments aren't entirely sent in to space. Certain fragments remain around the origin, others land up far from that. This paper considers only the sent fragments which characteristics are number, shape, mass, departure angles, and departure velocity (denominated "source terms" and considered as uncertain variables) (see Sect. 2). The fragments can impact the surrounding equipments. This possibility corresponds to the probability of impact, $P_{i m p}$ [1]. Next the authors write the fragment trajectory equations in $3 \mathrm{D}$ in order to study the impact problem between a fragment and an equipment (see Sect. 3). The Monte-Carlo simulation allows to determine the impact probability, $P_{i m p}$ (according to the source terms probability distributions). Thus the numerical simulations using data collected from the accident in Mexico City [2] have been performed. This study allows evaluating the fragment impact range and the arriving fragment energies at the impact on the surrounding equipments.

\footnotetext{
a Corresponding author:

Quoc-Bao.Nguyen@univ-paris-est.fr
}

\author{
Nomenclature \\ n fragment number, \\ $v \quad$ fragment velocity, \\ $m$ fragment mass, \\ $\varphi \quad$ vertical angle, \\ $\theta$ horizontal angle, \\ $D \quad$ drag force, \\ $L \quad$ lift force, \\ $C_{D} \quad$ drag coefficient, \\ $C_{L} \quad$ lift coefficient, \\ $S_{D} \quad$ frontal surface, \\ $S_{L} \quad$ projected surface to the ground, \\ $\rho_{\text {air }} \quad$ air density, \\ $P_{i m p}$ probability of impact.
}

\section{Threat}

\subsection{Fragment number, $n$}

According to an experimental study on cylindrical vessels [3-6], a crack initiates at circumferential welding and detaches one of end-caps, then propagates itself axially to unfold the ring of cylindrical vessel. On the other hand, the crack can also initiate at the middle of vessel because of the manufacture defects. It unfolds the ring, then propagates itself to the edges of the vessel, at last, following the circumference, detaches one or two end-caps. In two cases, the ring is either unfolded and remains at the origin, or it generates one or two fragments $[7,8]$. This explains why the quantity of plate is smaller than the one of end-cap and oblong end-cap (see Sect. 2.2). The fragment number 


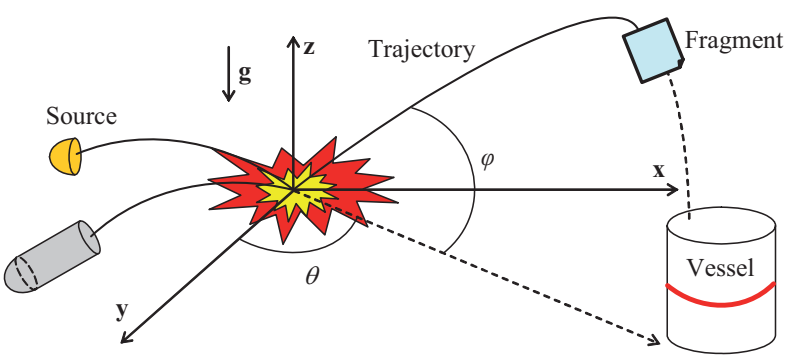

Fig. 1. Explosion generates fragment series.

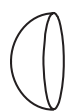

(a)

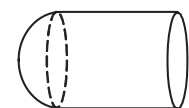

(b)

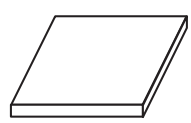

(c)
Fig. 2. Fragment shapes (a) end-cap; (b) oblong end-cap; (c) plate.

varies from one to three (rarely four). Simultaneously basing on the report of Holden [2], the authors determine the probability distribution for this variable while using the principle of maximum entropy [9]. It allows applying a discreet distribution for the fragment number that varies from one to four.

\subsection{Fragment shape}

As described previously, the fragment types are the endcap, the end-cap attached to completed or partial ring (the oblong end-cap) and the flattened piece of ring (the plate). Moreover, according to the accident report of Holden [2], the generated fragments due to an explosion are end-caps (44 pieces), plates (57 pieces) or oblong endcaps (86 pieces) (Fig. 2). In first approach, the end-caps represent $25 \%$ of generated fragments, $30 \%$ for the plates and $45 \%$ for the oblong end-caps.

\subsection{Fragment mass, $m$}

The crack can initiate at the circumferential welding, or at the middle of vessel (see Sect. 2.1). That allows applying a constant probability distribution for the oblong end-cap length and for the plate dimensions. When the shape, the size, as well as the constituent material of fragment are known, its mass is then determined.

\subsection{Vertical departure angle, $\varphi$}

With the hypothesis that the crack can initiate anywhere on the vessel, the vertical departure angle is situated in the interval $\left[-90^{\circ}, 90^{\circ}\right]$. Basing on this hypothesis, the vertical departure angle is supposed follow a constant distribution in the interval $\left[-90^{\circ}, 90^{\circ}\right]$.

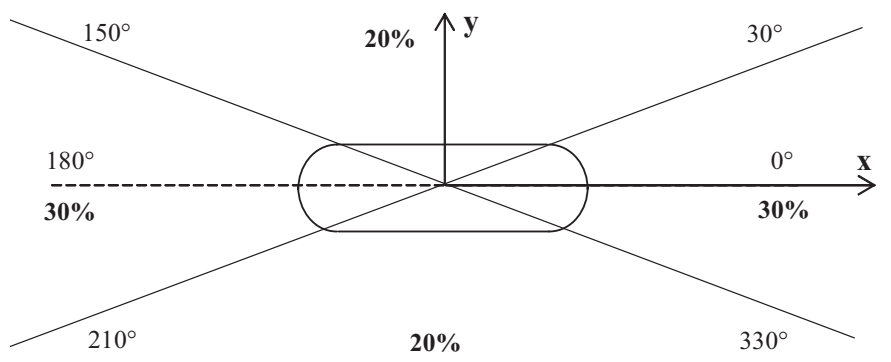

Fig. 3. Fragment distribution.

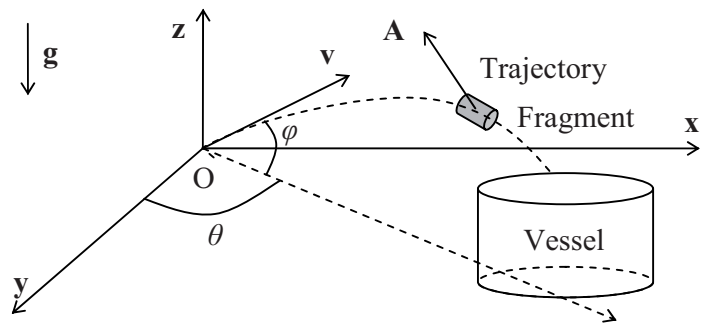

Fig. 4. Fragment trajectory.

\subsection{Horizontal departure angle, $\theta$}

Basing on the accident analysis $[2,7]$, a fragment distribution in relation to the principal vessel axis is carried out: 24 on 42 fragments are projected in an angular sector of $60^{\circ}$ around the principal axis and the remainder is projected in the perpendicular direction. An approximate distribution is considered for the horizontal departure angle: $20 \%$ of fragments in the interval $\left[30^{\circ}, 150^{\circ}\right] ; 30 \%$ of fragments in $\left[150^{\circ}, 210^{\circ}\right] ; 20 \%$ of fragments in $\left[210^{\circ}, 330^{\circ}\right]$ and $30 \%$ of fragments in $\left[330^{\circ}, 30^{\circ}\right]$ (Fig. 3).

Moreover a big number of end-caps and oblong endcaps is situated in the angular sector of the longitudinal vessel axis and of the perpendicular axis for the plate fragments. Nevertheless, some end-caps and oblong endcaps are found on the perpendicular direction and on the vessel axis for the plates. A possible interpretation of this phenomenon is the rotation of the end-caps, oblong endcaps and plates when they were cut [7].

\subsection{Departure velocity, $v$}

The departure velocity is determined while knowing its kinetic energy, $E_{c}$ and its mass, $m . E_{c}$ is deducted from the total energy, $E$ with a multiplying factor situated in the interval $[0.2,0.5]$ ( 0.2 is the advised value). This energy serves to expansion, to vessel rupture, to fragment kinematics, etc. and it is calculated by the equations of Brode, Baker and Baum [10]. On the other hand, a probabilistic study based on experiments of Baum [3,4] allows applying a log-normal probability distribution for the departure velocity, $v$. 


\section{Impact}

\subsection{Fragment trajectory}

During the flight, a fragment is subjected to the following forces:

- the weight $\overrightarrow{\mathbf{G}}=m \overrightarrow{\mathbf{g}}$, when $\overrightarrow{\mathbf{g}}$ is the acceleration of gravity vector;

- the aerodynamic force, $\overrightarrow{\mathbf{A}}$ is decomposed into two parts: the drag and the lift,

- the drag, $\overrightarrow{\mathbf{D}}$ is a combination of friction forces due to the air flow. It is parallel and opposed to the fragment trajectory

$$
D=\frac{1}{2} \rho_{a i r} v^{2} C_{D} S_{D}
$$

- the lift, $\overrightarrow{\mathbf{L}}$ is created by the aspiration in the depression zone. It is perpendicular to the fragment movement

$$
L=\frac{1}{2} \rho_{a i r} v^{2} C_{L} S_{L}
$$

In order to determine the impact point between a fragment and an equipment, these forces are projected on three principal axis $\overrightarrow{\mathbf{x}}, \overrightarrow{\mathbf{y}}, \overrightarrow{\mathbf{z}}$ including the horizontal and vertical angles $(\theta$ and $\varphi$, respectively):

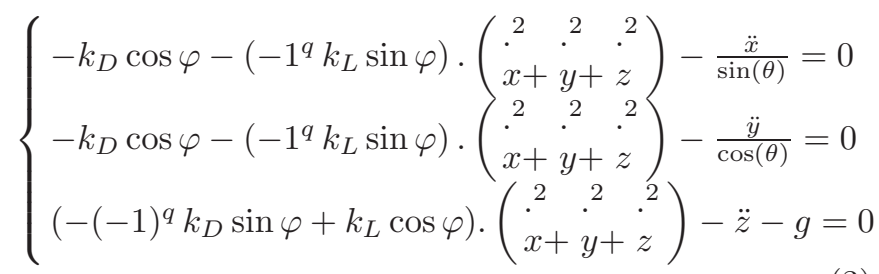

$$
k_{D}=\frac{1}{2} \frac{\rho_{a i r} C_{D} S_{D}}{m} ; \quad k_{L}=\frac{1}{2} \frac{\rho_{a i r} C_{L} S_{L}}{m}
$$

with $q=1$ for the descending; $q=2$ for the ascending; $x$, $y, z$, are the fragment center coordinates; $m$, the fragment mass; $C_{D}$, the drag coefficient; $S_{D}$, the frontal surface; $C_{L}$, the lift coefficient; $S_{L}$, the projected surface to the ground; $\rho_{\text {air }}$, the air density.

The lift and drag coefficients $\left(C_{L}\right.$ and $\left.C_{D}\right)$ are obtained experimentally [11]. In the first approach, the functions of the angle between the velocity and the fragment axis are created for each coefficient.

\subsection{Interaction}

The fragment shapes are the end-cap, the oblong endcap and the plate. The equipment shapes are cylindrical, spherical or cube. Previously, the impact problem between two ellipsoids $\left(\mathbf{E}_{1}, \mathbf{E}_{2}\right)$ is analyzed:

$$
\begin{array}{ll}
\left(\mathbf{E}_{1}\right) & \mathbf{X}^{\mathrm{T}} \mathbf{A X}=0 \\
\left(\mathbf{E}_{2}\right) & \mathbf{X}^{\mathrm{T}} \mathbf{B X}=0
\end{array}
$$

with $\mathbf{A}, \mathbf{B}$, their characteristic matrices; $f(\lambda)=$ $\operatorname{det}(\lambda \mathbf{A}+\mathbf{B})=0$, their characteristic equation; and $\mathbf{X}=(x, y, z, 1)^{\mathrm{T}}$.
Table 1. Data for the numerical simulations.

\begin{tabular}{cccc}
\hline \multicolumn{2}{c}{ Cylindrical vessel $(\mathbf{A})$} & \multicolumn{2}{c}{ Spherical vessel $(\mathbf{B})$} \\
\hline \multirow{2}{*}{ Radius } & $r_{x}=2 \mathrm{~m}$ & & $r_{x}=2 \mathrm{~m}$ \\
& $r_{y}=8 \mathrm{~m}$ & Radius & $r_{y}=8 \mathrm{~m}$ \\
Length & $r_{z}=8 \mathrm{~m}$ & & $r_{y}=8 \mathrm{~m}$ \\
Thickness & $0.07 \mathrm{~m}$ & Thickness & $0.05 \mathrm{~m}$ \\
\end{tabular}

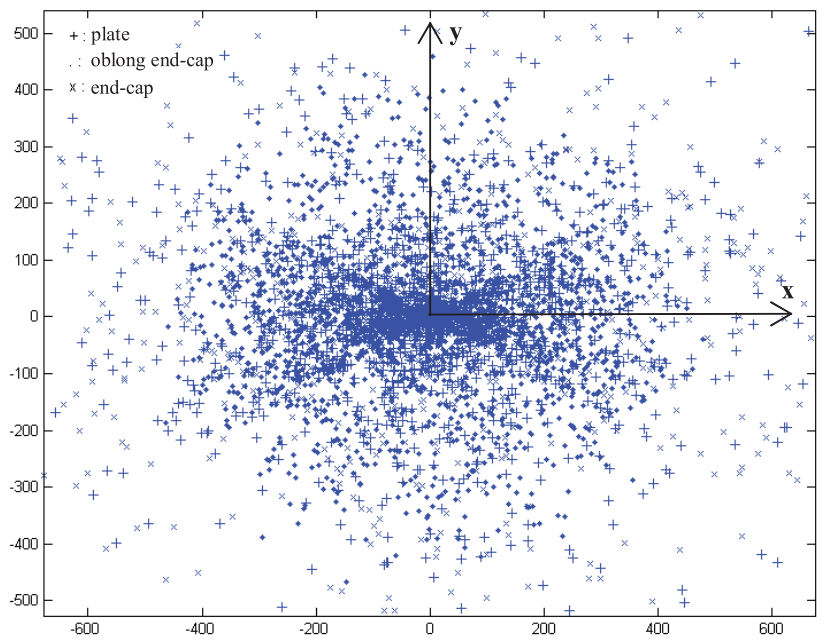

Fig. 5. Fragment distribution.

According to the results justified by Wang et al. [12]:

- the equation $f(\lambda)=0$ has always at least of two negative roots;

- two ellipsoids $\left(\mathbf{E}_{1}, \mathbf{E}_{2}\right)$ are separated by a plan if $f(\lambda)=0$ has two different positive roots;

- two ellipsoids $\left(\mathbf{E}_{1}, \mathbf{E}_{2}\right)$ intercept themselves if $f(\lambda)=$ 0 has a double positive root, $\lambda_{0}$.

In case of tangency, the intersection point, $\mathbf{X}$ is determined by solving equation (7):

$$
\left(\lambda_{0} \mathbf{I}+\mathbf{A}^{-1} \mathbf{B}\right) \mathbf{X}=0
$$

with $\mathbf{I}$, the matrix identity.

In a second time, the development of this model allows to obtain the impact condition between fragment and equipment.

\section{Numerical simulations}

Using the Monte-Carlo method, the numerical simulations are realized while considering two cylindrical vessels $\mathbf{A}$ and $\mathbf{B}$ with volumes equals to: $100 \mathrm{~m}^{3}$ and $2100 \mathrm{~m}^{3}$, respectively. The distance between them is about $20 \mathrm{~m}$. The data used to carry out these simulations are given in Table 1 and the results are presented in Figures 5-7.

One can notice (Fig. 5) that after explosion of vessel A (placed at the origin) the generated fragments are sent into a circular zone having a diameter equal to $1 \mathrm{~km}$ approximately. The majority of fragments is distributed in the angular sector of $60^{\circ}\left( \pm 30^{\circ}\right.$ in comparison with the 


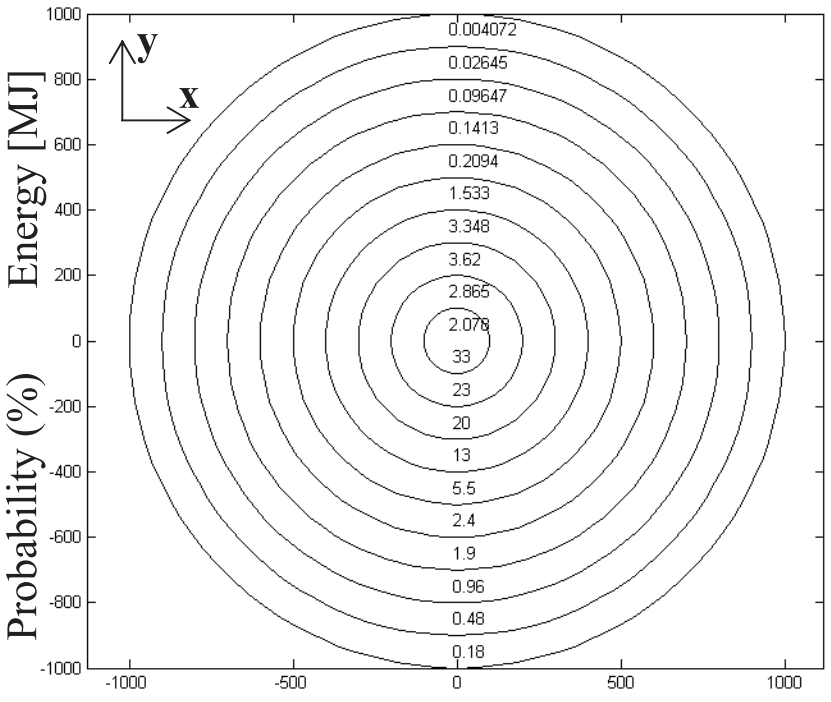

Fig. 6. Arriving fragment probability and their energy.

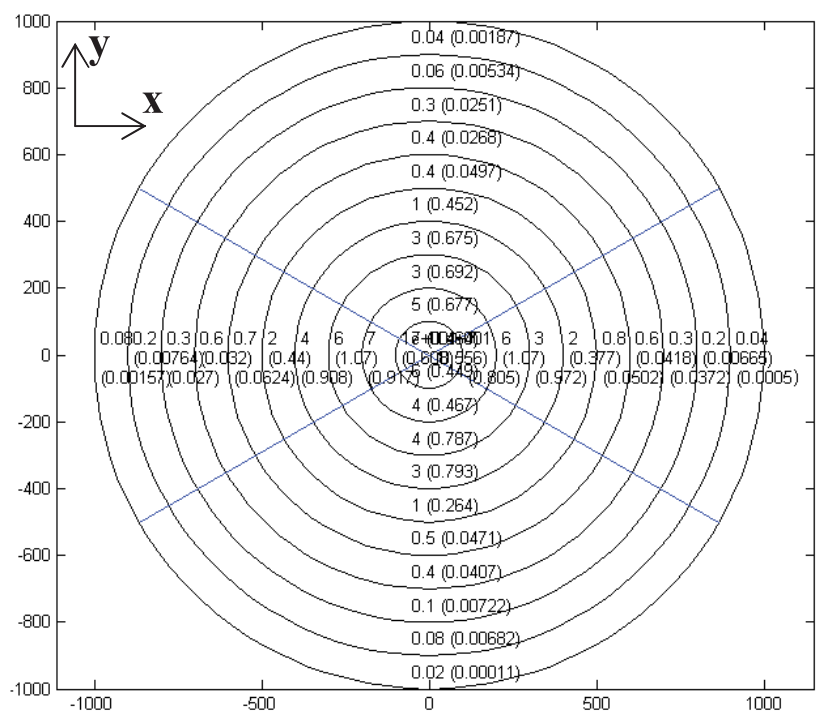

Fig. 7. Arriving fragment probability and their energy per sector. (The number between brackets represent energies in [MJ], the others correspond to the arriving projectile probabilities.)

axis $\overrightarrow{\mathbf{X}}$ of vessel $\mathbf{A}$ ). The probability of the arriving fragment energies are reported in Figures 6,7. One can notice that the last two quantities decrease according to the distance from origin. The arriving fragment velocity average is $150.19 \mathrm{~m} / \mathrm{s}$. The arriving fragment energy average is
35.06 MJ, the energy lost during movement is 6.50 MJ. The impact probability is $P_{i m p}=0.038 \%$.

\section{Conclusions}

The authors analyze the generated fragments reaches after a cylindrical vessel explosion. The fragment characteristics are considered as uncertain variables. A fragment movement model considering its shape and its size is then developed. This allows evaluating the impact probability, $\mathrm{P}_{i m p}$, between a fragment and an equipment in the industrial domain.

This research work has received the support of Institut National de l'Environnement Industriel et des Risques (INERIS), France.

\section{References}

1. A. Mébarki, R. Ami Saada, Q.B. Nguyen, F. Mercier, M. Reimeringer, Mechanical study integrated probabilistic approaches used to analyze the industrial risks, International Conference on Computational 8 Experimental Engineering and Sciences (Miami, USA, 0308 January 2007)

2. P.L. Holden, Assessment of Missile Hazards: Review of Incident Experience Relevant to Major Hazard Plant (Safety and Reliability Directorate, Health \& Safety Directorate, 1988)

3. M.R. Baum. J. Loss Prev. Process Ind. 8, 149 (1995)

4. M.R. Baum. J. Loss Prev. Process Ind. 12, 137 (1999)

5. M.R. Baum. J. Loss Prev. Process Ind. 14, 199 (2001)

6. Ineris, Les éclatements de réservoirs, Phénoménologie et modélisation des effets ( $\mathrm{N}^{\circ}$ Ineris-DRA-2004-46055, 2004)

7. Ineris, Calculs des effets mécaniques d'un BLEVE de citerne ferroviaire ( $\mathrm{N}^{\circ}$ Ineris-DRA-72293, 2005)

8. Q.B. Nguyen, A. Mébarki, F. Mercier, R. Ami Saada, M. Reimeringer, in Proceedings of the Eighth International Conference on Computational Structures Technology, edited by B.H.V. Topping, G. Montero, R. Montenegro (Civil-Comp Press, Stirlingshire, U K, 2006)

9. C. Soize, Probabilités et modélisation des incertitudes (2004)

10. U. Hauptmanns. J. Loss Prev. Process Ind. 14, 395 (2001)

11. P. Liu, X. Deng. J. Aéronautique et Spatial du Canada 49, 31 (2003)

12. W. Wang, J. Wang, M. Kim. Computer Aided Geometric Design 18, 531 (2001) 